\title{
The role of complement in experimental autoimmune myasthenia gravis
}

\author{
Linda L. Kusner ${ }^{1}$ and Henry J. Kaminski ${ }^{2}$ \\ ${ }^{1}$ Department of Pharmacology and Physiology, The George Washington University, Washington, \\ DC \\ 2Department of Neurology, The George Washington University, Washington, DC
}

\section{Abstract}

Complement plays an important role in the pathophysiology of experimental autoimmune myasthenia gravis (EAMG). The deposition of IgG at the neuromuscular junction, followed by the activation and observance of $\mathrm{C} 3$ at the site, and finally the insertion of the membrane attack complex, which results in the destruction of the plasma membrane at the neuromuscular junction. Animal models' of complement-deficient components show the importance of the mediated lysisin EAMG. These events have regulators that allow for the limitation in the cascade and the ability of the cell to inhibit complement at many places along the pathway. The complement regulatory proteins have many roles in reducing the activation of the complement cascade and the inflammatory pathways. Mice deficient in complement regulatory proteins, decay accelerating factor and CD59, demonstrate a significant increase in the destruction at the neuromuscular junction. Inhibition of complement-mediated lysis is an attractive therapeutic in MG.

\section{Keywords}

complement; complement regulators; myasthenia gravis; C5; autoimmunity

\section{Introduction}

Myasthenia gravis (MG) is an antibody-mediated autoimmune disease that targets the neuromuscular junction (NMJ). The preponderance of experimental data indicates that the deleterious effects of AChR autoantibody are mediated through activation of complement in animal models. Among this evidence, it has been observed that: $\mathrm{C} 3$ activation fragments, $\mathrm{C}$, and other components of the membrane attack complex (MAC) are readily detectable at motor endplates in animals with EAMG; ${ }^{1-3}$ depletion of $\mathrm{C} 3$ by cobra venom factor protects rats against induction of EAMG; ${ }^{45}$ and administration of complement inhibitors (anti-C6, anti-C5, soluble CR1, or rEV576) ${ }^{6-9}$ protects in passively induced and active EAMG. The data, taken together, strongly support the notion that complement activation in particular assembly of the MAC at the NMJ leads to destruction of post-synaptic structure, compromising neuromuscular transmission.

In human MG, there is strong evidence for complement as the primary pathogenic mechanism. This derives from identification of $\mathrm{C} 3$ and MAC deposition at NMJ of 
patients. ${ }^{2,3,10} \mathrm{NMJ}$ consistently demonstrate a simplified structure consistent with complement injury. ${ }^{11}$ Depletion of serum complement components, C3 and C4, is observed in patients, ${ }^{12}$ terminal components of complement are present in MG patient sera, ${ }^{13}$ and sera induces complement-mediated lysis of cultured myotubes. ${ }^{14}$ The role of complement in MG associated with antibodies directed towards the muscle specific kinase or LRP-4 has not been fully defined and is not discussed in this review.

\section{Complement}

Complement is a major defense in the innate immune system and is recognized as augmenting the adaptive immune response. The complement system is made up of over 30 proteins with the function to eliminate invading pathogens, prevent infection, and clear altered host cells. The initiation of complement can occur by three separate mechanisms: classical, alternative, and lectin pathways. Although the activation of the pathways differs, all intersect at the C3 level, and progress to the membrane attack complex (Fig. 1). In MG, the classical pathway is involved by the initiation of the antigen/antibody complex being recognized by $\mathrm{C} 1 \mathrm{q}$. The process progresses to the membrane attack complex (MAC) insertion into the postsynaptic membrane of the NMJ.

The initiation of the classical pathway by the binding of the antibody (IgG or IgM) to the antigen (AChR) is recognized by $\mathrm{Clq}$ (Fig. 1). The $\mathrm{Clq}$ forms a pentameric complex with the $\mathrm{C} 1 \mathrm{r} 2 \mathrm{C} 1 \mathrm{~s} 2$ serine protease tetramer. The conformational change of $\mathrm{C} 1 \mathrm{q}$ binding to the cell surface allows $\mathrm{C} 1 \mathrm{r}$ to activate through auto-cleavage. ${ }^{15}$ Active $\mathrm{C} 1 \mathrm{r}$ can then cleave $\mathrm{C} 1 \mathrm{~s}$. The zymogen reaction continues by $\mathrm{C} 1$ s proteolytic activation ofC4 to $\mathrm{C} 4 \mathrm{a}$ (peptide analphylatoxin), and $\mathrm{C} 4 \mathrm{~b}$ which attaches to the cell surface and binds $\mathrm{C} 2$. $\mathrm{C} 2$ also becomes cleaved by $\mathrm{C} 1$ s to form the $\mathrm{C} 3$ convertase $(\mathrm{C} 4 \mathrm{~b} 2 \mathrm{a})$. The $\mathrm{C} 3$ convertase cleaves $\mathrm{C} 3$ forming $\mathrm{C} 3 \mathrm{a}$ (peptide analphylatoxin) and $\mathrm{C} 3 \mathrm{~b}$, which binds the $\mathrm{C} 3$ convertase to become the $\mathrm{C} 5$ convertase (C4b2a3b). The activation of C5 by C5 convertase to form C5a and C5b initiates formation of the membrane attack complex by recruitment of $\mathrm{C} 6, \mathrm{C} 7, \mathrm{C} 8$, and $\mathrm{C} 9$.

\section{Complement-deficient EAMG models}

Animal models are efficient means of analyzing the effect of the complement system in MG. Animals can be induced in a passive form of EAMG with an antibody to the AChR or actively by administering the antigen. By these methods, the roles of the particular components of the complement system can be discriminated by producing NMJ damage and resultant weakness. Components to the complement system have been genetically deleted from mice and rats to discern the nature of the classical pathway and the relevance to the NMJ injury and antibody production.

Studies of complement depletion were the first approaches to assess the necessity of complement to the pathology of EAMG. Cobra venom was injected during the acute phase of the active EAMG in rats and before the passive transfer of antibodies to the AChR. In each experimental design, the lack of complement resolved the NMJ pathology that was seen in the complement sufficient groups. ${ }^{4}$ Direct evidence for the involvement of the classical complement pathway in EAMG derives from the identification of $\operatorname{IgG}, \mathrm{C} 3$, and MAC deposition at the neuromuscular junctions of both EAMG animals and MG patients. ${ }^{2}, 3,10,16,17$

Mice deficient in C3 and C4 demonstrate reduced clinical scores compared to their wildtype littermates during the course of active EAMG, with $\mathrm{C} 3$-knockout mice showing no signs of weakness. ${ }^{18}$ The total IgG and AChR specific IgG levels were equivalent in the C4deficient animals compared to their wild-type littermates, whereas, $\mathrm{C} 3$-deficient animals showed a reduction in total $\mathrm{IgG}$ and $\mathrm{AChR}$ specific IgG. Both C3-and C4-deficient animals 
demonstrate reduced AChR IgG2b levels. Reduced B cell expression was also seen in nonimmunized C3- and C4- deficient mice, which remained low in the EAMG state. Therefore, the state of the immune system is altered in the complement deficient animals. Despite the presence of AChR-specific antibodies neuromuscular junctions show an absence of $\mathrm{C} 3$ or MAC deposits when EAMG was induced in C3-deficient mice. Complement-deficient EAMG animals showed a preservation of AChR density at the neuromuscular junction.

A later component of the complement cascade, $\mathrm{C} 5$, also is involved in the development of EAMG. C5 cleavage by the C5 convertase results in C5a, a potent anaphylatoxin, and C5b, an initiator of MAC assembly. The influence of the C5 on EAMG susceptibility was analyzed in C5-sufficient and C5-deficient mice in an active model. ${ }^{19}$ During the time course of EAMG, both strains had comparable levels of serum AChR specific antibodies. C5-deficient mice demonstrated reduced incidence of disease compared to the C5-sufficient mice. Therefore, C5 appeared to be critical in EAMG pathogenesis, likely through activation of the terminal $\mathrm{C} 5 \mathrm{~b}-\mathrm{C} 9$ sequence required for neuromuscular junction destruction. As a potential for therapeutic target, blocking of C5 does not impair function of early activated complement components. ${ }^{7}$

The final components of the MAC involve $\mathrm{C} 5 \mathrm{~b}$ recruiting C6-C7-C8, which then accumulates $\mathrm{C} 9$, which forms the pose of the complex. A study on C6-deficient rats demonstrated that the lack of one of these components to the MAC causes resistance to passively induced EAMG.${ }^{17}$ The study also looked at the ability to restore the MAC complex and its destruction to the NMJ resulting in weakness by addition of C6 to a C6 deficient EAMG animal. Therefore, all components in the classical pathway must be in place for MAC deposition.

\section{Therapeutic targets in EAMG}

Since complement deficient animals demonstrated a resistance to EAMG, therapeutic agents that target the classical pathway of the complement system may allow improved outcome in MG. The classical pathway has many components that could be potential targets for inhibition of MAC and the reduction in the damage done to the NMJ in EAMG.

The target for therapeutic effect has focused on the C5 component. An antibody to C5 was used in a passive study on rats. ${ }^{7}$ The rats were given injections of anti-C5 or a control IgG four hours before being induced by AChR specific antibody. The IgG control and untreated rats showed weakness in 24 hours with untreated decreasing in strength over the time course. The rats treated with anti-C5 demonstrated only mild weakness on day 3, which resolved by day 5 . Complement deposition at the NMJ was assessed by immunohistochemistry to $\mathrm{C} 9$. The $\mathrm{C} 9$ is relatively absent at the $\mathrm{NMJ}$ of the anti-C5 treated group compared to the untreated and control IgG treated animals. The anti-C5 treated animal showed preserved synaptic folds of the NMJ, whereas, the untreated animals demonstrated reduced synaptic folds of the neuromuscular junction and increase in cell membrane debris.

A complement inhibitor has been discovered in the saliva of the soft tick. The compound was found to bind and modify C5, inhibiting its interaction with C5 convertase. ${ }^{20}$ To determine the effect of the complement inhibitor, labeled rEV576, a passive model of EAMG was employed in rats. ${ }^{6}$ Treatment withrEV576 demonstrated significantly less weakness compared to the untreated group. As part of the same study, the active model of EAMG was produced in Lewis rats. After weakness was observed, treatment was initiated with rEV576 or vehicle. The rats in the control group declined rapidly over the course of twelve days, whereas, the treated group improved. C9 deposition at the NMJ of the treated group was significantly less than the vehicle treated group. 


\section{Cell surface complement regulators}

The complement cascade has regulators, which are responsible for limiting damage of spontaneously activated complement. For the classical pathway of the complement cascade, decay accelerating protein (Daf, CD55), membrane co-factor protein (MCP, CD46), CR1related gene/protein Y (Crry), and CD59 are critical inhibitors of activation. These regulators have been found on the surface of most myofibers and concentrated at the NMJ. ${ }^{21,22}$ The CD59 inhibits the membrane attack complex formation and decay accelerating factor accelerates the decay of $\mathrm{C} 3$ convertase and $\mathrm{C} 5$ convertase. Both have been investigated in EAMG (see below).

\section{Levels of complement regulators vary among muscles}

The levels of complement regulators have been evaluated in diaphragm and extraocular muscle and found to change in response to passive EAMG induction. ${ }^{22}$ The transcript levels of Daf1, Crry, and CD59 were determined in the extraocular muscle and the diaphragm of C57BL/6 mice under normal and passive EAMG conditions. Under the normal condition, all three complement regulators' transcripts were lower in extraocular muscle compared to diaphragm. When comparing normal muscle to passive EAMG induced muscle, extraocular muscle had a reduction of the level of transcripts of the complement regulators during disease state. However, the diaphragm did not show any changes in complement regulators due to EAMG. In assessing the protein level, immunohistochemistry showed an increase in Daf1 expression at the NMJ of extraocular under EAMG conditions, whereas, EAMG induced showed an increase in Crry at the NMJ of the diaphragm. The study demonstrates a dynamic expression of complement regulators and the decrease expression may affect the susceptibility of muscles to complement mediated diseases.

The complement regulators have been assessed in the background of genetic modification of specific complement regulator expression. ${ }^{23}$ The transcript levels of Daf, CD59a, and $C D 59 b$ did not show any increases to compensate for the loss of the other complement regulator in genetically modified mice. However, the genetic knockout of both the Daf and CD59ab showed an increase in the Crry transcript levels. These results suggest that the modification of one of the complement regulators can affect the expression of other complement regulators. Also, disease and tissue type influence complement regulator levels.

\section{C9 deposition increases in complement regulator knockout mice with passive EAMG}

To determine the effect of EAMG on complement regulator knockouts, wild-type mice and mice deficient in Daf1, CD59ab, or both Daf1and CD59ab, were induced by McAb3 (gift of Dr. Vanda Lennon). ${ }^{23}$ After 24 hours, the Daf1 $1^{-/-} \mathrm{CD}_{59} \mathrm{ab}^{-/-}$mice required euthanasia due to the severity of weakness. The levels of MAC deposition was much greater in CD59ab deficient mice compare to wildtype and Daf $1^{-/-}$mice. The nature of MAC deposits differed among the Daf1 $1^{-/} \mathrm{CD} 59 \mathrm{ab}^{-/-}$mice having deposits internalized in the myofiber.

Ultrastructural analysis of the NMJ of the Daf1 $1^{-/}$CD59ab ${ }^{-/-}$animals demonstrated extensive damage at the NMJ as well as myofibers that were necrotic. Comparing only CD59ab deficient mice to the Daf1 $1^{-/}$mice, MAC deposition was much greater at the NMJ of the CD59ab ${ }^{-/-}$mice at 24 and 60 hours of EAMG induction. However, the Daf1 ${ }^{-/-}$mice appeared weaker. Both complement regulator-knockout animals had much more severe weakness and MAC deposition than wild-type mice. These and other studies ${ }^{24-26}$ signal the critical importance of complement regulation confined to the NMJ and point to the possibility that enhancing complement inhibition at the NMJ may be a successful form of therapy. 


\section{Increasing complement inhibitors to the NMJ as a therapeutic}

In light of the complement regulator studies, we have developed a therapeutic approach to concentrate complement inhibitors to the NMJ. In a preliminary study, we produced a compound consisting of the Daf regulatory region conjugated to a single chain antibody that binds the acetylcholine receptor. The construct was found to bind the AChR and demonstrated in vivo complement inhibitory function. ${ }^{27}$ In unpublished studies, we have demonstrated in vitro efficacy and importantly have not found evidence of compromise of neuromuscular transmission by the compound.

\section{Conclusion}

We have briefly summarized work performed in rodents that support a therapeutic benefit for EAMG produced by complement inhibition. The extensive studies of complement and its regulators in EAMG have led to consideration that therapies could be targeted against the complement system for human MG, and in fact, a phase 2 trial of eculizumab, which targets the $\mathrm{C} 5$ component, for generalized MG was recently completed. It is clear that complement inhibition is a fruitful path for therapeutic evaluation; however, greater elucidation of the complement mediated pathology of MG is required. The differential susceptibility and response of muscle groups will be critical to define further in order to determine whether complement inhibition may be of greater benefit to patients with ocular myasthenia compared to generalized MG. Thus far, the C5 step of complement activation has been a focus for inhibition, but MAC assembly may be more important. Finally, limiting the adverse effects of chronic complement inhibition will be critical to enhance the treatment benefit; one approach would be to develop agents that specifically concentrate to the NMJ.

\section{Acknowledgments}

Work contained in this review was supported by grants from the National Institutes of Health, (R24EY014837and R01EY013238 to HJK) and the Myasthenia Gravis Foundation of America (LLK).

\section{References}

1. Sahashi K, et al. Ultrastructural localization of immune complexes (IgG and C3) at the end-plate in experimental autoimmune myasthenia gravis. J Neuropathol Exp Neurol. 1978; 37:212-223. [PubMed: 147324]

2. Sahashi K, et al. Ultrastructural localization of the terminal and lytic ninth complement component (C9) at the motor end-plate in myasthenia gravis. J Neuropathol Exp Neurol. 1980; 39:160-172. [PubMed: 7373347]

3. Nakano S, Engel AG. Myasthenia gravis: quantitative immunocytochemical analysis of inflammatory cells and detection of complement membrane attack complex at the end-plate in 30 patients. Neurology. 1993; 43:1167-1172. [PubMed: 8170563]

4. Lennon VA, et al. Role of complement in the pathogenesis of experimental autoimmune myasthenia gravis. J Exp Med. 1978; 147:973-983. [PubMed: 206648]

5. Tsujihata M, et al. Effect of myasthenic immunoglobulin $\mathrm{G}$ on motor end-plate morphology. Journal of neurology. 2003; 250:75-82. [PubMed: 12527996]

6. Soltys J, et al. Novel complement inhibitor limits severity of experimentally myasthenia gravis. Ann Neurol. 2009; 65:67-75. [PubMed: 19194881]

7. Zhou Y, et al. Anti-C5 antibody treatment ameliorates weakness in experimentally acquired myasthenia gravis. J Immunol. 2007; 179:8562-8567. [PubMed: 18056404]

8. Biesecker G, Gomez CM. Inhibition of acute passive transfer experimental autoimmune myasthenia gravis with Fab antibody to complement C6. J Immunol. 1989; 142:2654-2659. [PubMed: 2703710] 
9. Piddlesden SJ, et al. Soluble complement receptor 1 (sCR1) protects against experimental autoimmune myasthenia gravis. J Neuroimmunol. 1996; 71:173-177. [PubMed: 8982117]

10. Engel AG, et al. Immune complexes (IgG and C3) at the motor end-plate in myasthenia gravis: ultrastructural and light microscopic localization and electrophysiologic correlations. Mayo Clin Proc. 1977; 52:267-280. [PubMed: 870771]

11. Selcen D, et al. Are MuSK antibodies the primary cause of myasthenic symptoms? Neurology. 2004; 62:1945-1950. [PubMed: 15184594]

12. Romi F, et al. The role of complement in myasthenia gravis: serological evidence of complement consumption in vivo. J Neuroimmunol. 2005; 158:191-194. [PubMed: 15589053]

13. Barohn RJ, Brey RL. Soluble terminal complement components in human myasthenia gravis. Clinical neurology and neurosurgery. 1993; 95:285-290. [PubMed: 8299285]

14. Ashizawa T, Appel SH. Immunopathologic events at the endplate in myasthenia gravis. Springer seminars in immunopathology. 1985; 8:177-196. [PubMed: 2413561]

15. Wallis R, et al. Paths reunited: Initiation of the classical and lectin pathways of complement activation. Immunobiology. 2010; 215:1-11. [PubMed: 19783065]

16. Engel AG, et al. Passively transferred experimental autoimmune myasthenia gravis. Sequential and quantitative study of the motor end-plate fine structure and ultrastructural localization of immune complexes (IgG and C3), and of the acetylcholine receptor. Neurology. 1979; 29:179-188. [PubMed: 571062]

17. Chamberlain-Banoub J, et al. Complement membrane attack is required for endplate damage and clinical disease in passive experimental myasthenia gravis in Lewis rats. Clin Exp Immunol. 2006; 146:278-286. [PubMed: 17034580]

18. Tuzun E, et al. Genetic evidence for involvement of classical complement pathway in induction of experimental autoimmune myasthenia gravis. J Immunol. 2003; 171:3847-3854. [PubMed: 14500686]

19. Christadoss P. C5 gene influences the development of murine myasthenia gravis. J Immunol. 1988; 140:2589-2592. [PubMed: 3356901]

20. Roversi P, et al. The structure of OMCI, a novel lipocalin inhibitor of the complement system. Journal of molecular biology. 2007; 369:784-793. [PubMed: 17445829]

21. Navenot JM, et al. Expression of CD59, a regulator of the membrane attack complex of complement, on human skeletal muscle fibers. Muscle Nerve. 1997; 20:92-96. [PubMed: 8995588]

22. Kaminski HJ, et al. Complement regulators in extraocular muscle and experimental autoimmune myasthenia gravis. Exp Neurol. 2004; 189:333-342. [PubMed: 15380483]

23. Kusner LL, et al. Cell Surface Complement Regulators Moderate Experimental Myasthenia Gravis Pathology. Muscle Nerve. 2012 In press.

24. Tuzun E, et al. Complement regulator CD59 deficiency fails to augment susceptibility to actively induced experimental autoimmune myasthenia gravis. J Neuroimmunol. 2006; 181:29-33. [PubMed: 17056125]

25. Morgan BP, et al. The membrane attack pathway of complement drives pathology in passively induced experimental autoimmune myasthenia gravis in mice. Clin Exp Immunol. 2006; 146:294302. [PubMed: 17034582]

26. Kaminski HJ, et al. Deficiency of decay accelerating factor and CD59 leads to crisis in experimental myasthenia. Exp Neurol. 2006; 202:287-293. [PubMed: 16859686]

27. Song $\mathrm{C}$, et al. Protective effect of scFv-DAF fusion protein on the complement attack to acetylcholine receptor: a possible option for treatment of myasthenia gravis. Muscle Nerve. 2012; 45:668-675. [PubMed: 22499093] 


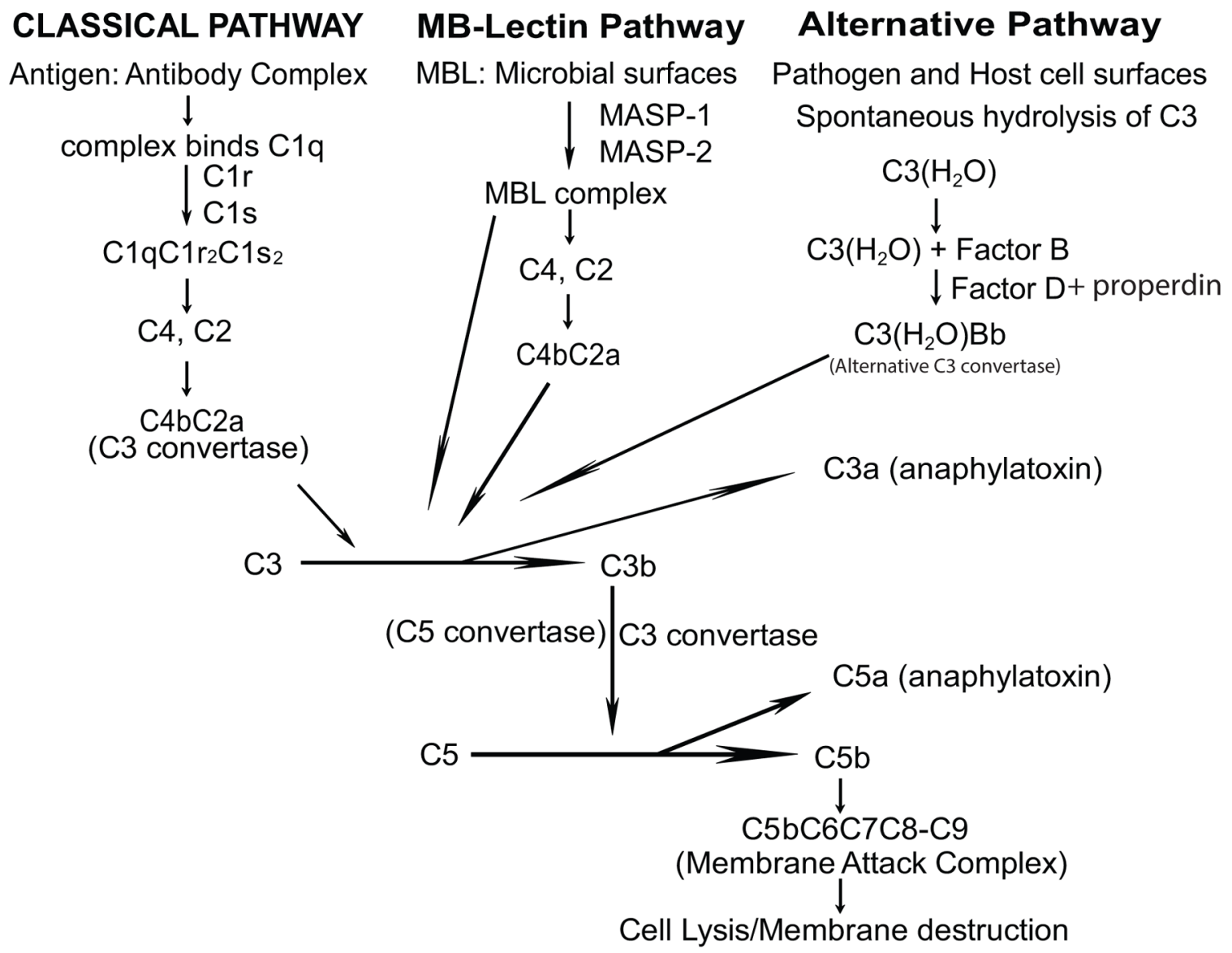

Figure 1.

Complement cascade. The three pathways of complement are initiated by different mechanisms and intersect at the C3 level. The classical pathway is involved in the pathology of EAMG, initiated by antibody-antigen complex. The final steps in the cascade are focused on the formation of the membrane attack complex, with $\mathrm{C} 5 \mathrm{~b}-\mathrm{C} 8$ accumulation on the plasma membrane and C9 recruitment to form the pore, which causes the destruction of the nervemuscle communication. 